\title{
La transnacionalización económica versus la autonomía de las políticas nacionales
}

\section{INTRODUCGIÓN}

Mucho se ha escrito sobre el proceso de internacionalización de la economía mundial. Là literatura referente a este tema se ha multiplicado en razón directa de la toma de conciencia del carácter internacional y sistemático de la crisis económica contemporánea y de la dimensión más comprehensiva de los problemas que están en la raíz de ambos fenómenos.

Naturalmente, existen aquellos que analizan este proceso de internacionalización desde una óptica que privilegia pretendidos aspectos lógicos y armoniosos, destacando una creciente integración de las estructuras productivas a nivel planetario y una interdependencia, también creciente, entre las naciones, como resultado de la evolución histórica mundial. Allí donde muchos destacaron la existencia de relaciones de dependencia y dominación entre las naciones, se podría verificar al contrario, de acuerdo con esta interpretación, la existencia de relaciones de interdependencia mutua, si bien correspondientes a países con distintos niveles de desarrollo de la base productiva. Bajo este prisma, Ia expansión en aumento de la base productiva industrial en escala planetaria correspondería a un proceso de progresiva homogenización de la estructura productiva: una especie de visión liberal neoclásica rediviva ${ }^{1}$ y una tendencia indudable en el mundo moderno.

En el otro extremo de la escala están aquellos que, al intentar una actualización histórica de la visión marxista originaria ${ }^{2}$, anali-

${ }^{1}$ Los países pobres, abundantes en mano de obra y con escasez de capital, tenderian a atraer a este último, en función de los bajos salarios reinantes. Los movimientos internacionales de capitales estarían asi orientados hacia esta área, lo que contribuiría más adelante a reducir los desniveles en los salarios reales.

${ }^{2}$ Así analizaban los autores del Manifiesto Comunista el proceso de exportación de capital: "al explorar el mercado mundial, la burgueśa dio un carácter cosmopolita a la producción y. al consumo de todos los países... en lugar de las antiguas necesidades satisfechas por la producción del país, surgen otras nuevas 
zan el fenómeno de la internacionalización como correspondiente a la gestación de un orden económico mundial unificado y sometido a una misma lógica, si bien diferenciada y desigual. Esta interpretación lleva a coincidencias en muchos puntos con el análisis anterior, a pesar de los enfoques diametralmente opuestos de los que parten.

Ambas posiciones están permeadas por múltiples enfoques de base analítica distinta. Su diversidad no disminuye, sino que por el contrario acrecienta la importancia del fenómeno. En realidad, la riqueza de las discusiones más recientes indica que tanto el proceso de internacionalización como los estudios que se producen sobre esos temas son parte, no sólo de la historia del análisis económico, sino que también se sitúan entre los temas que se localizan hoy en la frontera del conocimiento sobre la evolución económica.

\section{LA DIMENSIÓN EXISTÓRICA RECIENTE DEL PROCESO DE TRANSNACIONALIZAGIÓN}

A partir de la postguerra, cuando se da la conjunción de un período fuertemente expansivo de la economía mundial con la emergencia de las empresas multinacionales como artífices de la internacionalización del capital, se vuelve a colocar el proceso de internacionalización en el debate internacional. Este análisis está centrado en las características esenciales del fenómeno en el mundo capitalista, y el factor económico ha sido señalado consensualmente como el hilo conductor de la lógica del proceso, ya sea en los aspectos horizontales de la internacionalización - a través de la concurrencia y de la interpenetración de los bloques capitalistas constituidos por los principales centros desarrollados- ya sea a través de las relaciones de dominación y dependencia que caracterizan el sistema centro-periferia.

Por lo tanto, parece natural que relaciones económicas que vinculan entre sí a Ios países del llamado bloque socialista hayan sido dejadas de lado en los análisis efectuados sobre el fenómeno de transnacionalización, toda vez que son los factores políticos de dominación los que llevan claramente la primacía en esta área geopolítica. La transnacionalización asumía allí características menos "universales", en la medida en que la propia bipolaridad pre y postguerra fría limitaba rígidamente el espacio económico abarcado.

Es característico el hecho de que una ideología explícitamente

que reclaman, para su satisfacción, productos de las regiones y climas más distantes. En lugar del antiguo aislamiento y de la autarquía local y nacional, se introduce un tráfico ilimitado y la interdependencia de las naciones". Citado de Aníbal Pinto, La Internacionalización de la Economía Mundial y los Dilemas del Brasil, Unilivros, Río de Janeiro, 1981. 
internacionalista parecía constituir un lazo perfecto entre la amalgama política y económica prevaleciente, a pesar de que la reivindicación de un internacionalismo proletario no haya impedido la eclosión y el desarrollo de conflictos cada vez más abiertos. La Yugoslavia de Tito, ya a fines de la década de los 40 , representaba la primera resistencia abierta a la tentativa de integración internacionalizada (incluso en el ámbito de Europa Occidental), que se procuraba implementar. La tentativa de inserción de China en este esquema sólo resistió una década y estuvo cargada de conflictos y contradicciones. Rumania, Polonia y Checoslovaquia muestran hasta hoy las dificultades que alcanza este proceso, incluso dentro del cuadro limitado en que opera desde el punto de vista geográfico. Claro está que la pregunta válida es si se tendería a afirmar una integración internacionalizada de carácter circunscrito o si, en verdad, tiende cada vez más a ocurrir un proceso de desintegración transnacional, con la consiguiente reafirmación nacional.

Es un hecho incontestable el que el COMECON representa características especiales de un proceso de transnacionalización que, si bien no está acabado y está localizado en las dimensiones propias de un sistema imperial, presenta particularidades y especificidades de un fenómeno con raíces históricas más generales.

No se debe olvidar que el entrelazamiento que se verifica entre los países centralmente planificados y los países capitalistas desarrollados, por un lado, y también con los países del Tercer Mundo, por el otro, se da claramente en el marco del mercado capitalista mundial. Ello convierte este entrelazamiento en algo sujeto a la conocida lógica de mercado que regula este tipo de relaciones económicas internacionales.

En lo que se refiere al mundo capitalista, el mundo de postguerra, caracterizado por la vigorosa expansión productiva y del comercio a nivel global que dio origen a un verdadero boom de las inversiones privadas en el exterior, se vio reforzado por la emergencia de empresas transnacionales como agentes propulsores del proceso de internacionalización que ocurre dentro del marco del crecimiento dependiente. El fenómeno ha sido profusamente estudiado y ya existe hoy una literatura que puede ser considerada clásica, tanto sobre los temas de internacionalización del capital y de la economía mundial en este período como sobre la acción específica de las empresas transnacionales ${ }^{3}$.

Como testigo de este interés a nivel internacional, se puede destacar el hecho de que las Naciones Unidas han sido llevadas a formar un grupo de "personas eminentes" para estudiar el impacto de las Corporaciones Multinacionales sobre el Desarrollo y sobre las Relaciones Internacionales, organizando además un centro específi-

sVer nota bibliográfica al final. 
co para el estudio y la documentación sobre las empresas multinacionales:

En realidad, el orden político-económico mundial que se establece bajo la hegemonía norteamericana en la postguerra -la llamada "pax americana"- deja demarcado un cuadro extremadamente favorable al movimiento de expansión capitalista internacionalizado. Los Estados Unidos, que dominan en el campo militar y están en condiciones de asegurar la presencia de sus fuerzas esstratégicamente dislocadas por el mundo, ven, en la contraparte del pläno financiero, que el dólar ocupa el papel de moneda universal. Como también es indiscutible la hegemonía que los Estados Unidos ejercen en el plano industrial, comercial y tecnológico, el país èstá en condiciones de expandir avasalladoramente sus inversiones directas, procurando disfrutar de la fuerza de trabajo capacitada y de los mercados potenciales para los productos industriales, transformando en semiperiferia los diversos mercados nacionales de los países de Europa Occidental. Razones políticas y estratégicas, además de las económicas, hacen posible que en este periodo se realice una fuerte redistribución de los recursos financieros, fundamentalmente a través del Plan Marshall, posibilitando la reconstrucción económica de Europa Occidental que se torna, por consiguiente, un espacio económico privilegiado para las inversiones del capital industrial norteamericano.

La recuperación y el posterior b.oom de la economía de Europa Occidental, facilitadas por este flujo de inversiones directas; tienen su contrapartida en la consiguiente reacción que se establece a partir de la recuperación industrial verificada. De esta manera, los países europeos occidentales, especialmente Alemania, y a su Iado el Japón, -curiosamente los dos principales derrotados en la Segunda Guerra Mundial- pasan entonces a disputar una tajada creciente del mercado mundial que se expande rápidamente.

Una característica fundamental de este período es el inequívoco predominio de las relaciones Norte-Sur, o intercéntricas, sobre läs centro-periferia capitalistas, aunque éstas estén establecidas y en expansión. En otras palabras, el desarrollo y la profundización de la división internacional del trabajo ocurrió, preferencialmente a través del intercambio comercial y financiero que liga las economías industrializadas ${ }^{4}$.

Esto no significa que los países periféricos hayan pasado a lo largo de este proceso más global de internacionalización. Por el contrario, la penetración de sus economías nacionales por los agentes de la transnacionalización - las empresas multinacionales- se produce también en forma acelerada, principalmente en aquellos países que poseen un atractivo especial por la dimensión de su mercado interno.

${ }^{+}$A. Pinto, Op. Git. 
Así, a la articulación horizontal entre las economías desarrolladas se yuxtapone una articulación vertical Centro-Periferia (NorteSur). La primera se caracteriza por la concentración y centralización del capital productivo, en una fase inicial, y por el desarrollo de la conglomeración y diversificación oligopólica de las empresas transnacionales en los sectores productivos. La segunda presenta niveles diferenciados en función del privilegiamento estratégico : (casos de Taiwán, Corea, etc.), dimensión del mercado interno (Brasil, México) o disponibilidad de recursos minerales estratégicos (Venezuela, Irán).

Las empresas transnacionales son agentes privilegiados de este proceso de transnacionalización global; ya en 1957, según estudios efectuados a partir de levantamientos realizados por el Departamento de Comercio norteamericano, un cuarto de la producción mundial total estaba internacionalizada. Un estudio de las Naciones Unidas para el año 1971 constataba que, tomando un conjunto de 7.276 empresas matrices y sus 27.300 filiales, éstas respondían por cerca de un quinto del valor total del producto bruto mundial (excluidos los países de planificación centralizada) ${ }^{6}$.

La crisis del petróleo encontrará en el auge de su dinamismo al proceso de internacionalización, señalando entonces la afirmación de la renacida presencia de un nuevo componente fundamental: el capital financiero. La gestación de un circuito financiero internacionalizado, impulsada por el persistente déficit de la balanza de pagos norteamericana y por el desarrollo del mercado de euro-dólares en la década de los 60, termina por madurar en los años 70, a partir de la necesidad de financiamiento de las grandes inversiones internacionales $\mathrm{y}$ del déficit acumulado por los países importadores de petróleo, junto a la nueva misión resultante del proceso de intermediación bancaria relacionada con la urgente necesidaci del reciclaje de los petrodólares.

La contrapartida de esta expansión del sistema financiero internacional está representada por la extraordinaria aceleración del proceso de endeudamiento externo de Ios países subdesarrollados. En lo que se refiere a las operaciones en el mercado de euromonedas, por ejemplo, estos países, según el Morgan Guaranty Trust, pasan de una participación de $9,3 \%$ en 1970 a $51,2 \%$ entre 1975 y 1977 y aproximadamente a $57,4 \%$ en $1979 .{ }^{7} \mathrm{El}$ propio mercado de euromo-

"Estudio del Prof. Polk, en S. Hymer, "Le Imprese Multinazionali", Einaudi, Torino, 1971, citado por Massimo Micarelli en el artículo "Internazionalizzazione del Capitale e Multinazionali", Polftica Internazional, Nis 10-11, 1978.

'Naciones Unidas, Las Corporaciones Multinacionales y el Desarrollo Mundial, 1973.

${ }^{7}$ World Financial Markets, Morgan Guaranty Trust, 1979; Financial Market Trends, OECD, febrero de 1980. 
nedas no cesa de expandirse aceleradamente, pasando de un volumen de 57 billones de dólares en 1969 a casi 230 billones en 1975 y 650 billones de dólares en junio de 1980. A fines de 1979, la deuda acumulada por los países del Tercer Mundo con los bancos comerciales occidentales llegaba por sí sola a 149 billones de dólares, en cuanto a la de los miembros de COMECON les correspondía 77 billones ${ }^{8}$.

La creciente participación del sistema bancario transnacional en el financiamiento de los déficit en cuenta corriente de los países en desarrollo va colocando una serie de limitaciones y restricciones al margen de maniobra de sus políticas económicas. Las previsiones de una expansión continuada del mercado de empréstitos internacionales por los bancos privados no alejan, sino que por el contrario estimulan las especulaciones sobre el riesgo creciente de un colapso financiero internacional de graves proporciones ${ }^{9}$.

\section{IMPAGTO SOBRE LAS ECONOMÍAS NAGIONALES Y SOBRE LAS POSIBILIDADES DE FORMULAGIÓN DE POLÍTICAS AUTÓNOMAS: \\ ALGUNOS HECHOS Y PROPOSICIONES DE UNA AGENDA. DE INVESTIGAGIÓN}

El proceso de creciente internacionalización (integración) de los mercados y de la producción a nivel de la economía mundial, descrito de manera muy sumaria en la sección anterior, tiene evidentemente un profundo impacto sobre las economías nacionales, que se expresa no sólo en la introducción de nuevos patrones de producción y consumo que le son inherentes, sino que también se refleja a nivel de la distribución de renta generada entre las naciones, por un lado, y en el grado de autonomía en la formulación de políticas propias que le es permitido a cada país, por otro lado.

En el caso de los países periféricos, la nueva dinámica introducida por las empresas transnacionales tiene como resultado el establecimiento de una nueva división internacional del trabajo, al cual permite un cierto tipo de crecimiento, que Fernando Henrique Cardoso llama apropiadamente el "desarrollo asociado". El mismo Cardoso hace notar en este proceso de industrialización dependiente asociado la reproducción, en otro plano, de las asimetrías estructurales entre las economías centrales periféricas ya destacadas en los

\footnotetext{
${ }^{8}$ Para las últimas cifras, ver Prospects, 1981/1. Swiss Bank Corporation; Survey, enero de 1981, F. M. I.

"Ver en particular el esclarecedor artículo de Samuel Lichtensztejn, "De la crisis al colapso financiero internacional", en Economía de América Latina, CIDE, No 5, 1980. El Seminario de IRI y CEESTEM - México, sobre "Procesos de Endeudamiento Externo en América Latina", realizado recientemente (agosto de 1981) en la PVC, Río de Janeiro, dejó señaladas diversas contribuciones analíticas importantes para el dimensionamiento real del problema.
} 
análisis de la escuela de dependencia: "... aunque sea verdad que la actual fase de industrialización mundial requiere la dispersión en escala mundial de parte del sistema productivo, los fondos de acumulación continúan siendo retenidos en forma centralizada y el desarrollo de nuevos procesos y técnicas productoras se hace monopólicamente en el Centro. En consecuencia, la relación entre las economías industrializadas del Centro y de la Periferia es "interdependiente" pero "asimétrica"10.

Presenta incluso obvias insuficiencias la tentativa de promocion de algunos países a un status superior con el establecimiento de categorías como la de los NIC's (Newly Industrialized Countries), en base a procurar una diferenciación entre los países periféricos. No sólo demuestran estos países una inserción congénitamente deformada en el sistema económico internacional, por la propia superposición entre los sectores modernos e internacionalizados de sus economías y aquellos "atrasados", en una reproducción de un ambiguo dualismo de vasos comunicantes. Además, se mantienen como importadores líquidos de capital financiero y tecnologías, sin el control de los cuales no pueden asegurar una base autónoma para la generación e implementación acelerada de nuevas técnicas productivas y la garantía de sus relaciones económicas a nivel mundial.

No se trata de negar los resultados industrializantes de la transnacionalización una vez que, al posibilitar la transferencia hacia la periferia de parte del proceso productivo, la propia expansión de las empresas multinacionales comienza a necesitar y requerir tanto de sus nuevos mercados cuanto de su mano de obra. Si exceptuamos los casos de las zonas libres de producción (y en algunos casos de las llamadas "plataformas de exportación" como Singapur y Taiwán), que sólo representan una reproducción de lo que se llamó "economías de enclave" en el período agro-minero-exportador tan conocido en América Latina, es la expansión del mercado interno la que permite la absorción de la creciente producción de bienes de consumo durables y livianos, como también del desarrollo relativo del sector de bienes de capital. No se puede ignorar el hecho de que incluso cuando opera simplemente como abastecedor de las empresas internacionales instaladas en el territorio nacional, el sector local termina por quedarse con una parte del facturamiento del sector. En el caso de la industria automotriz brasileña, por ejemplo, las empresas nacionales de piezas para autos facturan aproximadamente un tercio de las ventas totales del sector, a pesar de que las empresas armadoras son de propiedad extranjera ${ }^{11}$.

${ }^{10}$ Fernando Henrique Gardoso, "Interdependência e Desenvolvimiento", conferencia ante el II Encuentro Inter-Regional sobre Desarrollo, Investigación, Co. municación y Educación, promovido por GLACso y OECD, Bogotá, junio de 1979.

"Dercio Garcla Munhoz, "Capitais Estrangeiros - a espera do Pragmatismo", en Revista de ANPEC, No 2, 1978. 
Entretanto, el padrón de producción que se transmite a la periferia lleva también consigo la reproducción de padrones de consumo que abren espacio a la marginalización de inmensos sectores de la población -como los trabajadores rurales y aquellos localizados en el sector informal urbano-y, además, a la incorporación desigual y deformada de los propios sectores de las clases trabajadoras que están vinculados al modo de producción internacionalizado, transformados así, como dice María da Conceição Tavares, en consumidores de segunda clase. Sin embargo, la expansión del mercado interno que la propia transnacionalización requiere y promueve, posibilita que varios segmentos de la sociedad obtengan ventajas con la industrialización ya alcanzada, y esto no sólo a nivel del sector empresarial y técnico gerencial. El propio proletariado industrial puede beneficiarse de la relativa diseminación de las ganancias obtenidas, en la med̦ida en que la misma organización del mercado de trabajo imponga esta condición en que los trabajadores demuestren capacidad de lucha.

En el curso de este proceso se establecen las condiciones para una nueva articulación entre la burguesía nacional, las empresas transnacionales y el Estado. En las palabras de Cardoso, la función política de la burguesía se redefine: "Ias multinaciones se asocían subordinadamente en el proceso de acumulación global. Luchan y se agitan mucho buscando apoyo en el Estado. Es tanto, el empresariado local lo rechaza, cuando éste avanza, cumpliendo su función de soporte general de la acumulación y, es por consiguiente, regulador y protector de las empresas locales y también de las multinacionales. A su vez, el Estado invierte en áreas dinámicas, aumentando contradictoriamente su peso en la economía, ya que al mismo tiempo que estimula el sector privado compite con él. Por consiguiente, la nueva articulación económica unió el crecimiento del mercado interno al dinamismo de las empresas mültinacionales y al sector estatal" 12 .

Dentro de esta triple articulación, muy poco se conoce sobre los objetivos y condiciones de las políticas de asociación explícita del Estado con el capital privado nacional y extranjero, tal como se expresa en el llamado tri-partido ${ }^{13}$.

Como se sabe, este esquema propicia la emergencia de una "nueva especie de empresa" que deja de lado la división tradicional de "áreas de competencia" de inversionistas públicos y privados, de acuerdo al tipo de producto. Esta nueva política, al permitir el alejamiento de los padrones tradicionales de actuación de los inversionistas extranjeros al condicionar su participación a la asociación

I2F. H. Cardoso, Op. Cit.

${ }^{13}$ Modelo de asociación adoptado, por ejemplo, en el ámbito de las empresas del complejo petroquínico de Camaçari, en Bahía. 
Mauricio Dias D. / La transnacionalización económica versus la autonomfa ...

con el Estado brasileño y con capitales nacionales privados, dejó establecido un cuadro diferente para las relaciones entre las partes contratantes. Los éxitos y debilidades de esta política, las causas y factores determinantes de su fracaso cuando éstos ocurrieron, la evaluación de la transferencia real de tecnología en función de lo programado y de lo efectuado efectivamente, las ventajas y desventajas del modelo en comparación con las formas tradicionales de inversiones, son campos aún abiertos al análisis y que demandan una investigación más minuciosa.

El análisis del proceso de transnacionalización como una nueva dimensión del capital articulado en un sistema productivo a escala mundial14 abre también espacio a la definición de una amplia gama temática que debería ser desarrollada. Esta veta por investigar comprende desde el análisis de los aspectos estrictamente económicos que determinan la morfologia del proceso de "mundialización" - (esto es, de integración en el proceso productivo del capital mundial) y las formas de estructuración específica que este proceso asume país a país y región a región, tanto al nivel de los Estados Nacionales cuanto al de su articulación con los diferentes segmentos sociales a nivel local.

La concentración del poder económico a escala transnacional, a partir de la creciente integración de los mercados y de la producción de los países industrializados capitalistas, posibilita que se desarrollen, a nivel de la economía mundial, centros de decisión con capacidad de acción global cada día más autónomos con relación con los Estados Nacionales. En el conflicto entre las áreas de decisión que competen a los Estados Nacionales y al espacio cada vez más autónomo que estos centros de decisión transnacionales tienden a reivindicar en función de las actividades económicas que se van internacionalizando, surgen no pocas aristas de difícil superación. - Las tentativas de coordinación al estilo del establecimiento de un código de conducta para las corporaciones multinacionales, por ejemplo, o han sido de lenta y difícil gestación o parecen condenadas a la inocuidad de las fórmulas híbridas. En la medida en que este propio conflicto parece como no resuelto y tendiendo a agudizarse y a generalizarse merece, entre las investigaciones prioritarias, un estudio más particularizado de su actual alcance y tendencia a la ampliación.

Un hecho relacionado con este último punto se refiere a la simultaneidad entre el proceso de transnacionalización económica y la internacionalización a nivel del proceso político ${ }^{15}$, en que esta últi-

14El capital mundial correspondería a la forma de capital que se vincula a un sistema productivo que se realiza asimismo en la totalidad del mundo capitalista, en la definición de Herbet. de Souza (yer nota bibliográfica):

${ }^{10} \mathrm{EI}$ Seminario Internacional sobre "La. Internacionalización de la Política en 
ma se alimenta y es impulsada por la primera, tanto a nivel de la influencia de los factores internacionales en la política nacional cuanto en la "supernacionalización" cada vez más acentuada, a nivel de la elaboración y también de la difusión, de políticas y mecanismos de coordinación que buscan una mayor estabilización del sistema global donde actúan los agentes transnacionalizados.

En lo que se refiere a la transnacionalización de los circuitos firiancieros, el avance en el conocimiento de los marcos en que opera y a los que se sujeta ha sido mayor, a partir de su propio impacto sobre las economías latinoamericanas. Un rápido balance del conjunto de estudios ya realizados y del "estado de las artes" en esta área indica la urgente necesidad de una mayor integración y articulación de las investigaciones ya realizadas o en curso, principalmente en el marco de los estudios comparativos ${ }^{16}$. Además de la consolidación a nivel histórico de los estudios sobre el tema de las deudas externas (que, como se sabe, en el caso de la deuda pública de los países latinoamericanos acompaña la evolución de los Estados Nacionales desde su formación en el siglo xix), se vuelve importante analizar las dimensiones y formas actuales del problema, cuya gravedad al nivel de las repercusiones en el plano económico nacional son cada vez más evidentes. Paralelamente, a nivel global, la acumulación de deudas gigantescas contraídas por los países deudores vuelve a colocar en el centro de las discusiones sombrías predicciones de la inminencia del colapso financiero internacional ante una deuda que, por su dimensión, no podría ser pagada sin ingentes sacrificios económicos y sociales ${ }^{17}$.

La vigorosa expansión del mercado financiero internacional privado que se presentó en los últimos años, muestra țambién un campo abierto a las exploraciones, que contribuyen a revelar sus implicaciones a nivel de las economías nacionales y de la deuda pública y privada acumulada. Un aspecto que está adquiriendo relieve últimamente es la discusión del impacto que ocasiona esta creciente privatización o "bancarización"18 de las fuentes de financiamiento externo a nivel de la autonomía de las políticas nacionales. ¿En qué medida el volumen alcanzado por el endeudamiento externo impone restricciones reales al margen de maniobra de que disponen

América Latina", organizado en septiembre de 1981 por IRI, IUPERJ y FLACSO, intentó discutir ampliamente este fenómeno, en especial no "Internacionalización de la Política de la Economía", que reunió a investigadores del IRI y del cEEsTEM. ${ }^{16}$ Un esfuerzo en esta área correspondió al Seminario de Trabajo sobre "Endeudamiento Externo en América Latina", promovido en agosto de 1981 por el IRI/PUG/RJ Y por el GEESTEM.

${ }^{17}$ Sobre este último punto, ver en especial el artículo de Samuel Lichtensztejn, citado anteriormente.

${ }^{18}$ En la expresión usada por Rosario Green, del ceestem y del Colegio de México, en sus estudios sobre el tema. 
Mauricio Dias D. / La transnacionalización económíca versus ḷ autonomía... .

los gobiemos nacionales en la formulación de sus políticas "internas"? No se trata únicamente de la verificación de la capacidad de manipulación e imposición de condicionamientos a las políticas económicas nacionales acreedoras de los gobiernos locales, que de por sí son bástante acentuadas. También se verifica el grado de autodisciplina y control que establece una especie de autocensura por iniciativa propia, al internalizar el gobierno políticas propiciadas por los organismos financieros internacionales privados y multilaterales ${ }^{19}$.

Tampoco se pueden dejar de analizar los conflictos que presenta la llamada "apertura al exterior", en sus aspectos de vinculación con el circuito financiero internacional, en lo que se refiere a la autonomía nacional en las determinaciones de estrategias de desarrollo para cada país comprometido, así como las restricciones en términos de las opciones y prioridades en las áreas económica y social. Este campo de análisis requiere, además, mucho trabajo de profundización analítica, en especial en lo que se refiere a la conjunción de las restricciones estrictamente económicas con las de orden más específicamente político.

Se trata, por ejemplo, de entender las articulaciones a nivel internacional que garantizan la reproducción de un orden político favorable a la transnacionalización económica. Por ejemplo, ¿en qué medida pueden los bancos privados negociar "objetivamente" con el Estado brasileño, cuando dos tercios de la deuda externa del Brasil (que alcanzará a 70 billones de dólares a fines de 1981) son de su responsabilidad? Esa es una materia importante para la investigación y el modus operandi de estos organismos, así como de las empresas transnacionalizadas, en la evaluación del riesgo político de empréstitos e inversiones de un cierto país. ¿Cómo se determina el llamado "riesgo país" en la evaluación de los grandes bancos internacionales? ¿Cuál es la función del spread como factor de ponderación subjetivo y también como criterio objetivo? ¿Cómo estos organismos procuran influir en la vida política y económica de los paises con que se relacionan?20 Aunque ello pueda ocurrir en determinados casos, no se trata de acusar a las corporaciones multinacionales $y$ a los integrantes de los circuitos financieros transnacionalizados de una acción dilatoria determinada a priori, en que los fenómenos señalados impliquen un debilitamiento del Estado Nacional. Es interesante estudiar la creciente dificultad para establecer políti-

19Pedro S. Malan llama la atención sobre este problema en el caso brasileño en el trabajo "Problema da Divida Externa de Brasil: Una nota para Discussão", presentado al Seminario TR/CEEESTEM.

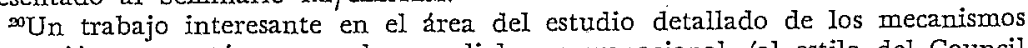
de presión que actúan a escala mundial o supranacional (al estilo del Council of Foreign Affairs) ha sido desarrollado por Rene Dreyfuss y Bill Smith, de Ia UEMG. 
cas nacionales autónomas en un contexto en el cual, cada vez más frecuentemente, las decisiones tomadas fuera del espacio económico y político regido por el Estado Nacional pasan a reflejarse en él como una fuerza incontenible.

Se derivan adicionalmente de estas dificultades graves problemas de coordinación, una vez que, saliendo de los cuadros clásicos de las estructuras de poder nacional, la autonomía de las empresas transnacionales con relación al Estado produce situaciones conflictivas no sólo en los planos político y económico sino también en el social. En las palabras de Celso Furtado, "es evidente que proseguir por el camino de la internacionalización de las economías significa aceptar la institución de centros de decisión con poderes para titular el conjunto del sistema capitalista, aunque esa tutela se limite a la definición de directrices de política económica y social, radicando en cada gobierno la responsabilidad de traducirlas en reglas operativas"21 ¿Cuáles son las implicaciones más concretas de este proceso? Cuáles son sus proyecciones a corto y mediano plazo? ¿Podrá este proceso ser aceptado como algo inevitable e inelúdible por todas las fuerzas sociales y políticas aceptadas?

Es el ámbito de las propuestas para la estructuración de un nuevo orden económico internacional, estas cuestiones han sido relegadas a un segundo plano. Más allá de la actitud de oposición apriorística de los que ven las proposiciones del diálogo Norte-Sur ya sea como una utopía, ya sea como una miopía de los países involucrados, las cuestiones en juego tienen dimensiones que merecen un esfuerzo conjugado de investigación para ser realizado a nivel internacional. Por ejemplo, se pueden tomar las propuestas para elevar las transferencias financieras desde los países industrializados hacia los países en desarrollo, o la propuesta para un "Nuevo Plan Mrundial de Empleos", formulada por un conjunto de economistas, entre ellos Tinbergen, como una forma de estimular la economía mundial.

Se trata, pues, de un conjunto de problemas amplio y diversificado, que por su trascendencia e impacto, merece una redoblada atención por parte de estudiosos y analistas. La descripción preliminar y necesariamente sucinta que aquí hemos presentado, sólo pretende facilitar la colocación en la mesa de discusiones de una agenda de investigaciones, aunque sea embrionaria.

No se pretende crear una nueva categoría profesional, la de los "transnacionalistas". Pero, parece claro que en el ámbito de las Giencias Sociales, que son interdisciplinarias, existen herramientas teóricas suficientes para abordar los problemas enfocados en forma esclarecedora. Y tanto en la Economía como en la Política, del mismo modo que en las buenas historias policiales, la cuestión no está

${ }^{21}$ Celso Furtado, “O Brasil Pós-Milagre”, Paz e Terra, Río, 1981. 
Mauricio Dias D. / La transnacionalización económica versus la autonomła...

sólo en saber quién es el principal responsable, sino, también, el porqué de los acontecimientos y la lógica y racionalidad de su dinámica propia.

\section{NOTA BIBLIOGRAFICA}

1. De una manera selectiva y necesariamente reducida, podemos citar entre dos estudios clásicos: Barnett, R. y Muller, R., Global Reach '(Nueva York, Simon and Schuster, 1974); Palloix, G., Las firmas intemacionales y el proceso de internacionalización. (México, DF: Siglo xxr, 1975); Hymer, S., "Empresas Multinacionais: A Internacionalizaçao do Capital" (Rlo Graal, 1978); Hymer, S., "The Efficiency (Contradictions) of Multinational Corporations", American Economic Review, 60 (1970) y "The Multinational Corporation and the Law of Uneven Development" en J. N. Bahagwati, org. Economics and World Order (New York, Mac Millan, 1972); Hymer, S. y Rowthorn, R., International Big Business. 1957/1967 (London: Cambridge U. Press, 1971); Fajnzilber, F. et al., Las Empresas Multinacionales en América Latina (Buenos Aires: Edit. Periferia, 1973); Fajnzilber, F. y Martínez Tarragó, T., Las empresas transnacionales: Expansión a nivel mundial y proyección en la industria mexicana (México, DF: Fondo de Cultura Económica, 1976) ; Furtado, C., "Post National
Capitalism", LARU Studies, II 2 (1978); "The Internationalization of Production", World Development, (1973) ; Continho, L. y Belluzzo, L. $G_{4}$, "O Desenvolvimento do Capitalismo Avancado e a Reorganização da Economia Mundial no Pós-Geurra", Estudos CEBRAP, 23 (1979); Newfarmer, R. S. y Mueller, W. F., Multinational Corporations in Brazil and Mexico, Report to the Subcommittee on Foreign Relations (1975) .

2. Se trata del artículo de Herbert de Souza, "El Concepto de Capital Mundial", en Estados Unidos: Perspectiva Latinoamericana, CIDE, N9 8, 29 semestre de 1980.

3. Un texto para la discusión recientemente preparado por María de Conçêicao, Tavares presenta una excelente contribución al entendimiento de la internacionalización. del capital y sus efectos en la economia brasileña. Se trata de Tavares, M. C. y Teixeira, A., "A Internacionalização do Capital e as Mutinacionais na Indústria Brasileira", Texto para Discusión No 1, FEA/UFRJ, 1981. 